Y. MUTŌ

KODDAI MATH. SEM. REP

26 (1975), 409-423

\title{
CRITICAL RIEMANNIAN METRICS ON PRODUCT MANIFOLDS
}

\author{
By Yosio Mutō
}

A critical Riemannian metric of this paper means a critical point of a functional $I$ of a $C^{\infty}$ Riemannian metric $g$ on a compact orientable $C^{\infty}$ manifold $M$, restricted by $\operatorname{Vol}(M, g)=1$ and defined by an integral of the square of the curvature tensor of $(M, g)$. In the present paper a critical Riemannian metric $g_{12}$ such that $\left(M, g_{12}\right)=\left(M_{1}, g_{1}\right) \times\left(M_{2},{ }^{\prime} g_{2}\right)$ is studied and relations between critical Riemannian metrics $g_{12}, g_{1}$ and $g_{2}$ on $M, M_{1}$ and $M_{2}$ respectively are obtained. Furthermore it is shown that in certain cases the index of $I$ at $g_{12}$ is positive.

In a previous paper [5] the present author considered the space $\mathscr{M}(M)$ of $C^{\infty}$ Riemannian metrics $g$ on a compact orientable $C^{\infty}$ manifold $M$ satisfying the condition

$$
\int_{M} d V_{g}=1
$$

where $d V_{g}$ is the volume element of $M$ measured by $g$. He studied a mapping $I: \mathscr{M}(M) \rightarrow \boldsymbol{R}$ induced by the integral

$$
I[g]=\int_{M}\left\|K_{g}\right\|^{2} d V_{g}
$$

where $K_{g}$ is the curvature tensor of $(M, g)$ and $\left\|K_{g}\right\|^{2}$ is its square.

If $\eta$ is a diffeomorphism of $M$ and $\eta^{*}$ its pull back, then we have $\eta^{*}(g) \in \mathscr{M}(M)$ and $I[g]=I\left[\eta^{*}(g)\right]$. Let $\mathscr{D}(M)$ be the group of diffeomorphisms of $M$ and $\mathscr{M}(M) / \mathscr{D}(M)$ be the space where each point is an orbit $O_{g}$ by $\mathscr{D}(M)$ through an element $g$ of $\mathscr{M}(M)$. Then we can deduce a mapping $\widetilde{I}: \mathscr{M}(M) / \mathscr{D}(M) \rightarrow \boldsymbol{R}$ from the mapping $I: \mathscr{M}(M) \rightarrow \boldsymbol{R}$ by $\widetilde{I}\left(O_{g}\right)=I[g]$. As $O_{g}$ is a critical point of $\widetilde{I}$ if and only if $g$ is a critical point of $I$, we adopt the convention to say that $g$ is a critical point of $\widetilde{I}$ when $O_{g}$ is a critical point of $\widetilde{I}$. We also say that $\widetilde{I}$ has a minimum or a local minimum at $g$ when $\tilde{I}$ has a minimum or a local minimum at $O_{g}$. Thus, if we say that $\tilde{I}$ has a local minimum at $g$, this means that there exists a neighborhood $U$ of $O_{g}$ in $\mathscr{M}(M)$ such that, if $g_{1}$ is a Riemannian metric satisfying $g_{1} \oplus O_{g}, g_{1} \in U$, then $I\left[g_{1}\right]>I[g]$.

Remark 1. The manifold of $C^{\infty}$ Riemannian metrics on $M$, which we denote for the present by $\mathscr{M}^{\#}(M)$ in order to distinguish from our $\mathscr{M}(M)$, has been

Received Nov 26, 1973. 
studied by D. Ebin [4]. He has analysed the action of $\mathscr{D}(M)$ on $\mathscr{M}^{\#}(M)$ and proved the existence of submanifolds $S$ of $\mathscr{M}^{\#}(M)$ with a certain property. It results that to study deformations in $\mathscr{M}^{\#}(M) / \mathscr{D}(M)$ we need only study curves in $\mathscr{M}^{\#}(M)$ whose tangent at $g$ is in $\delta^{-1}(0)$, namely, orthogonal to the orbits (see also M. Berger and D. Ebin [3]). The same is also valid with $\mathscr{M}(M)$ as the latter is a submanifold of $\mathscr{M}^{\#}(M)$ invariant by the action of $\mathscr{D}(M)$.

Remark 2. The mapping $I$ has been studied by $M$. Berger and the formula for a critical point has been obtained [2].

It was proved in [5] that, when $M$ is diffeomorphic to an $S^{n}, \widetilde{I}$ has a local minimum at a metric $g_{0}$ of positive constant curvature.

The purpose of the present paper is to study the mapping $I$ or $\tilde{I}$ when $M$ is a product manifold $M_{1} \times M_{2}$ where $M_{1}$ and $M_{2}$ are compact orientable $C^{\infty}$ manifolds.

When we say in the present paper that $g$ is a critical Riemannian metric on $M$, it always means that $g$ is a critical point of the mapping $I$ or $\widetilde{I}$ defined by $(0,2)$. At that time $(M, g)$ is called a critical Riemannian manifold.

First we get the following theorems.

THEOREM 1. Let $M, M_{1}, M_{2}$ be compact orientable $C^{\infty}$ manifolds such that $M=M_{1} \times M_{2}$ and $\operatorname{dim} M_{1}=m_{1}, \operatorname{dim} M_{2}=m_{2}$. Let $g_{12} \in \mathscr{M}(M)$ be a $C^{\infty}$ Riemannian metric such that there exist a Riemannian metric ' $g_{1}$ homothetic to a critical Riemannian metric $g_{1}$ on $M_{1}$ and a Riemannian metric ' $g_{2}$ homothetic to a critical Riemannian metric $g_{2}$ on $M_{2}$ satisfying

$$
\left(M, g_{12}\right)=\left(M_{1}, ' g_{1}\right) \times\left(M_{2}, ' g_{2}\right) .
$$

Then a necessary and sufficient condition that $g_{12}$ be a critical Riemannian metric on $M$ is that the square $\left\|^{\prime} K_{1}\right\|^{2}$ of the curvature tensor of $\left(M_{1}, g_{1}\right)$ and the square $\left\|^{\prime} K_{2}\right\|^{2}$ of the curvature tensor of $\left(M_{2}, ' g_{2}\right)$ be constant and

$$
\frac{\left\|^{\prime} K_{1}\right\|^{2}}{m_{1}}=\frac{\left\|^{\prime} K_{2}\right\|^{2}}{m_{2}} \text {. }
$$

THEOREM 2. Let $M, M_{1}, M_{2}$ be compact orientable $C^{\infty}$ manifolds and let $g_{12} \in \mathscr{M}(M)$ be such that there exist a Riemannian metric ' $g_{1}$ on $M_{1}$ and a Riemannian metric ' $g_{2}$ on $M_{2}$ satisfying

$$
\left(M, g_{12}\right)=\left(M_{1}, ' g_{1}\right) \times\left(M_{2}, g_{2}\right) .
$$

Then a necessary and sufficient condition that $g_{12}$ be a critical Riemannian metric is that ' $g_{1}$ and ' $g_{2}$ be homothetic to a critical Riemannian metric $g_{1}$ on $M_{1}$ and a critical Riemannian metric $g_{2}$ on $M_{2}$ respectively and the squares of the curvature tensors, $\left\|^{\prime} K_{1}\right\|^{2}$ and $\left\|^{\prime} K_{2}\right\|^{2}$, of the Riemannian manifolds $\left(M_{1},{ }^{\prime} g_{1}\right)$ and $\left(M_{2},{ }^{\prime} g_{2}\right)$ respectively be constant satisfying

$$
\frac{\left\|^{\prime} K_{1}\right\|^{2}}{m_{1}}=\frac{\left\|^{\prime} K_{2}\right\|^{2}}{m_{2}}
$$


In the last part of the present paper, the index of $I$ at such critical Riemannian metric $g_{12}$ is studied and it is proved that this index is positive in certain cases. Especially the mapping $I: \mathscr{M}\left(S^{m_{1}} \times S^{m_{2}}\right) \rightarrow \boldsymbol{R}$ has positive index at a critical Riemannian metric $g_{12}$ such that $\left(S^{m_{1}} \times S^{m_{2}}, g_{12}\right)=\left(S^{m_{1}},{ }^{\prime} g_{1}\right) \times\left(S^{m_{2}},{ }^{\prime} g_{2}\right)$ where ${ }^{\prime} g_{1}$ and ' $g_{2}$ are Riemannian metrics of positive constant curvature, if $m_{1} \geqq 3$ and $m_{2} \geqq 3$, or, if $m_{1} \geqq 4$ and $m_{2}=2$. This is a remarkable result as A. Avez has obtained the following theorem [1].

THEOREM A. Let $M$ be a compact orentable $C^{\infty}$ manifold of dimension 4. Then the functional $I[g]$ has an absolute minimum at $g$ if and only if $g$ is an Einstein metruc.

\section{$\S 1$. Product manifold and Riemannian metrics.}

Let $M, M_{1}, M_{2}$ be compact orientable $C^{\infty}$ manifold such that $M=M_{1} \times M_{2}$ and let $\mathscr{M}(M)$ be the space of all $C^{\infty}$ Riemannian metrics $g$ on $M$ such that the volume of $M$ measured by $g$ is 1 . Similarly we can define $\mathscr{M}\left(M_{1}\right)$ and $\mathscr{M}\left(M_{2}\right)$. Let us consider a Riemannian metric $g_{12} \in \mathscr{M}(M)$ such that there exist Riemannian metrics ' $g_{1}$ and ' $g_{2}$ satisfying $\left(M, g_{12}\right)=\left(M_{1},{ }^{\prime} g_{1}\right) \times\left(M_{2},{ }^{\prime} g_{2}\right)$ where ' $g_{1}$ and ' $g_{2}$ need not satisfy ' $g_{1} \in \mathscr{M}\left(M_{1}\right)$, or ' $g_{2} \in \mathscr{M}\left(M_{2}\right)$. We denote the set of all such Riemannian metrics $g_{12}$ by $\mathscr{M}_{12}\left(M_{1} \times M_{2}\right)$ or $\mathscr{M}_{12}(M)$.

To begin with we calculate the curvature tensor of $\left(M, g_{12}\right)$.

Let $U_{\xi}, \xi \in \Lambda_{1}$, and $V_{\eta}, \eta \in \Lambda_{2}$, be coordinate neighborhoods of $M_{1}$ and $M_{2}$ respectively such that $\left\{U_{\xi}, \xi \in \Lambda_{1}\right\}$ and $\left\{V_{\eta}, \eta \in \Lambda_{2}\right\}$ cover $M_{1}$ and $M_{2}$ respectively. Then $\left\{U_{\tilde{\xi}} \times V_{\eta}, \xi \in \Lambda_{1}, \eta \in \Lambda_{2}\right\}$ covers $M$ and we can use local coordinates

$$
\left(x_{(\xi)}{ }^{1}, \cdots, x_{(\tilde{\xi})}^{m_{1}}, y_{(\eta)}{ }^{m_{1}+1}, \cdots, y_{(\eta)}{ }^{m_{1}+m_{2}}\right) \text {, }
$$

where $m_{1}=\operatorname{dim} M_{1}, m_{2}=\operatorname{dim} M_{2}$, to denote a point $P=P_{1} \times P_{2}$ of $M$ if $P \in U_{\tilde{\xi}} \times V_{\eta}$.

We let the indices $a, b, c, \cdots, h, \imath, j, \cdots, p, q, r, \cdots$ run the range $\left\{1, \cdots, m_{1}\right\}$ and the indices $\alpha, \beta, \gamma, \cdots, \kappa, \lambda, \mu, \cdots, \pi, \rho, \sigma, \cdots$ the range $\left\{m_{1}+1, \cdots, m_{1}+m_{2}\right\}$. We also let the indices $A, B, C, \cdots, H, I, J, \cdots, P, Q, R, \cdots$ run the range $\{1, \cdots$, $\left.m_{1}, m_{1}+1, \cdots, m_{1}+m_{2}\right\}$ so that a point of $M$ may be denoted by $\left(x_{(\tilde{\xi})}{ }^{h}, y_{(\eta)}{ }^{\kappa}\right)$ or simply by $\left(x^{h}, y^{\kappa}\right)$. Moreover, $\left(x^{\Delta}\right)$ stands for $\left(x^{h}, y^{\kappa}\right)$. We use natural frame in each coordinate neighborhood $U_{\xi} \times V_{\eta}$ so that a tensor is expressed by its components. For example, a $(1,1)$-tensor of $M$ is given by $T_{B}{ }^{A}$ or, if written separately, by $T_{b}{ }^{a}, T_{\beta}{ }^{a}, T_{b}{ }^{\alpha}, T_{\beta}{ }^{\alpha}$.

Since $M$ is a product manifold and the local coordinates in $M$ are induced by local coordinates in $M_{1}$ and those in $M_{2}$, a $(1,1)$-tensor field $A_{b}{ }^{a}$ on $M_{1}$ and a $(1,1)$-tensor field $B_{\beta}{ }^{\alpha}$ on $M_{2}$ induce a $(1,1)$-tensor field $C_{B}{ }^{A}$ on $M$ such that

$$
C_{b}{ }^{a}(P)=A_{b}{ }^{a}\left(P_{1}\right), \quad C_{\beta}{ }^{\alpha}(P)=B_{\beta}{ }^{\alpha}\left(P_{2}\right), \quad C_{\beta}{ }^{\alpha}(P)=C_{b}{ }^{a}(P)=0
$$

where $P=P_{1} \times P_{2}$. But in general a $(1,1)$-tensor field $T_{B}{ }^{A}$ on $M$ does not have such a property, for example, $T_{b}{ }^{a}(P)$ may depend on $y^{\kappa}$ and $T_{\beta}{ }^{a}$ need not vanish.

Now, let $g_{12} \in M_{12}(M)$ be a Riemannian metric on $M$ such that 


$$
\left(M, g_{12}\right)=\left(M_{1}, g_{1}\right) \times\left(M_{2}, g_{2}\right) .
$$

Denoting the components of $g_{12}, g_{1},{ }^{\prime} g_{2}$ by $g_{J I},{ }^{\prime} g_{j i}, g_{\mu \lambda}$ respectively, we have

$$
g_{j i}={ }^{\prime} g_{j \imath}, \quad g_{\mu \lambda}={ }^{\prime} g_{\mu \lambda}, \quad g_{j \lambda}=0 .
$$

Let $\left\{\begin{array}{l}H \\ J I\end{array}\right\}, '\left\{\begin{array}{l}h \\ j i\end{array}\right\}, '\left\{\begin{array}{c}\kappa \\ \mu \lambda\end{array}\right\}$ be the Christoffel symbols derived from $g_{J I}, ' g_{j i}, ' g_{\mu \lambda}$ respectively and $K_{K J I}{ }^{H},{ }^{\prime} K_{k j i}{ }^{h},{ }^{\prime} K_{\nu \mu \lambda}{ }^{\kappa}$ be the components of the curvature tensors of $\left(M, g_{12}\right),\left(M_{1}, g_{1}\right),\left(M_{2},{ }^{\prime} g_{2}\right)$ respectively. Then we have

and

$$
\left\{\begin{array}{c}
h \\
j i
\end{array}\right\}=\left\{\begin{array}{c}
h \\
j i
\end{array}\right\}, \quad\left\{\begin{array}{c}
\kappa \\
\mu \lambda
\end{array}\right\}=\left\{\begin{array}{c}
\kappa \\
\mu \lambda
\end{array}\right\}, \quad \text { all other }\left\{\begin{array}{c}
H \\
J I
\end{array}\right\}=0
$$

$$
K_{k j i}{ }^{h}=^{\prime} K_{k j i}{ }^{h}, \quad K_{\nu \mu \lambda \lambda^{k}}=^{\prime} K_{\nu \mu \lambda \lambda^{k}}, \quad \text { all other } K_{K J I}{ }^{H}=0 .
$$

The covariant components $K_{K J I H},{ }^{\prime} K_{k j i h},{ }^{\prime} K_{\nu \mu \lambda \kappa}$ and the contravariant components $K^{K J I H},{ }^{k j i n},{ }^{\nu \mu \lambda \lambda \kappa}$ also satisfy

$$
\begin{array}{lll}
K_{k j i n}={ }^{\prime} K_{k j i h}, & K_{\nu \mu \lambda \kappa}={ }^{\prime} K_{\nu \mu \lambda \kappa}, & \text { all other } K_{K J I H}=0, \\
K^{k j i n}={ }^{\prime} K^{k j i n}, & K^{\nu \mu \lambda \kappa}={ }^{\prime} K^{\nu \mu \lambda \kappa}, & \text { all other } K^{K J I H}=0 .
\end{array}
$$

We have also

$$
\begin{array}{lll}
K_{j i}={ }^{\prime} K_{j i}, & K_{\mu \lambda}={ }^{\prime} K_{\mu \lambda}, & \text { all other } K_{J I}=0, \\
K^{j i}={ }^{\prime} K^{j i}, & K^{\mu \lambda}={ }^{\prime} K^{\mu \lambda}, & \text { all other } K^{J I}=0
\end{array}
$$

for the components of Ricci tensors of $\left(M, g_{12}\right),\left(M_{1},{ }^{\prime} g_{1}\right)$ and $\left(M_{2},{ }^{\prime} g_{2}\right)$. The scalar curvature $S c(K)$ of $g_{12}$ and the scalar curvatures $S c\left({ }^{\prime} K_{1}\right)$ of ' $g_{1}, S c\left({ }^{\prime} K_{2}\right)$ of ' $g_{2}$ satisfy

$$
S c(K)=S c\left({ }^{\prime} K_{1}\right)+S c\left({ }^{\prime} K_{2}\right) .
$$

Then we have the following formula for the integral $I\left[g_{12}\right]$,

$$
\begin{aligned}
I\left[g_{12}\right] & =\int_{M} K_{K J I H} K^{K J I H} d V_{g} \\
& =\int_{M}\left[{ }^{\prime} K_{k j i h}{ }^{\prime} K^{k j i n}+{ }^{\prime} K_{\nu \mu \lambda \kappa}{ }^{\prime} K^{\nu \mu \lambda \kappa}\right] d V_{g}
\end{aligned}
$$

where

$$
d V_{g}=\left\{\operatorname{det}\left({ }^{\prime} g_{j i}\right) \operatorname{det}\left({ }^{\prime} g_{\mu \lambda}\right)\right\}^{\frac{1}{2}} d x^{1} \cdots d x^{m}, \quad m=\operatorname{dim} M .
$$

\section{§ 2. A critical Riemannian metric on a product manifold (Proof of Theorem 1).}

Now we want to get a critical Riemannian metric $g_{12}$ on $M=M_{1} \times M_{2}$ such that $g_{12} \in M_{12}(M)$. 
The metric $g_{12}$ in (1.1) satisfies $g_{12} \in \mathscr{M}(M)$, while ' $g_{1}$ and ' $g_{2}$ need not satisfy $' g_{1} \in \mathscr{M}\left(M_{1}\right),{ }^{\prime} g_{2} \in \mathscr{M}\left(M_{2}\right)$. But it is easy to see that there exist some positive numbers $\alpha_{1}$ and $\alpha_{2}$ such that $g_{1}=\left(\alpha_{1}\right)^{2} g_{1}$ and $g_{2}=\left(\alpha_{2}\right)^{2 \prime} g_{2}$ satisfy $g_{1} \in \mathscr{M}\left(M_{1}\right)$ and $g_{2} \in \mathscr{M}\left(M_{2}\right)$. Let us find a relation between $\alpha_{1}$ and $\alpha_{2}$. If we denote for the present the volume element of $M$ measured by $g_{12}$ by $d V$ and the volume elements of $M_{1}$ and $M_{2}$ measured by ' $g_{1}$ and ' $g_{2}$ respectively by $d^{\prime} V_{1}$ and $d^{\prime} V_{2}$, then we have $d V=d^{\prime} V_{1} d^{\prime} V_{2}$, hence

$$
\left[\int_{M_{1}} d^{\prime} V_{1}\right] \cdot\left[\int_{M_{2}} d^{\prime} V_{2}\right]=1
$$

On the other hand, if we denote the volume elements of $M_{1}$ and $M_{2}$ measured by $g_{1}$ and $g_{2}$ respectively by $d V_{1}$ and $d V_{2}$, then we have

$$
\begin{aligned}
& \left(\alpha_{1}\right)^{m_{1}} \int_{M_{1}} d^{\prime} V_{1}=\int_{M_{1}} d V_{1}=1, \\
& \left(\alpha_{2}\right)^{m_{2} \int_{M_{2}} d^{\prime} V_{2}}=\int_{M_{2}} d V_{2}=1 .
\end{aligned}
$$

Hence we have

$$
\left(\alpha_{1}\right)^{m_{1}}\left(\alpha_{2}\right)^{m_{2}}=1
$$

A necessary and sufficient condition that a Riemannian metric $g$ be a critical Riemannian metric was obtained by M. Berger [2] as a system of differential equations involving the curvature tensor, the Ricci tensor, covariant derivatives of the scalar curvature and the Ricci tensor. Let us examine the equations for a moment.

For that purpose let $M$ be for the present any compact orientable $C^{\infty}$ manifold. If, using local coordinates $x^{1}, \cdots, x^{n}$ and the natural frame, we denote tensors by their components, so that the curvature tensor and the Ricci tensor by $K_{k j i}{ }^{h}$ and $K_{j i}$, and raise or lower indices by the components $g^{j i}$ or $g_{j i}$ of the fundamental tensor, the equations in question are as in [5]

$$
\begin{array}{rl}
2 \nabla_{j} \nabla_{i} & S c(K)-4 \nabla_{p} \nabla^{p} K_{j i} \\
& +4 K_{\jmath p} K^{p}{ }_{i}-4 K_{\jmath q p i} K^{q p} \\
& -2 K^{r q p}{ }_{j} K_{r q p i}+\frac{1}{2} K_{d c b a} K^{d c b a} g_{j i}=c g_{j i}
\end{array}
$$

where $S c(K)$ is the scalar curvature, $\nabla_{\imath}$ means the covariant differentiation with the use of the Christoffel symbols of $g$, and $c$ is a number which is chosen suitably so that a solution may exist.

Let us assume $g$ is a critical Riemannian metric and ' $g$ is a Riemannian metric homothetic to $g$, namely, there exists a positive number $\alpha$ such that $g=\alpha^{2} g$. Let the components of ' $g$ be denoted by ' $g_{j i}$ and the components of the curvature tensor and the Ricci tensor of $\left(M,{ }^{\prime} g\right)$ by ${ }^{\prime} K_{k j i}{ }^{h}$ and ' $K_{j i}$. Let the indices of these tensors be raised and lowered by the components ' $g^{j i}$ and ' $g_{j i}$ 
of the fundamental tensor ' $g$ and the scalar curvature of $(M, ' g)$ be denoted by $S c(' K)$. As $g$ and ' $g$ have the same Christoffel symbols, covariant differentiation is the same in $\left(M,{ }^{\prime} g\right)$ as in $(M, g)$ and we have ' $K_{k j i}{ }^{h}=K_{k j i}{ }^{h},{ }^{\prime} K_{j i}=K_{j i}, S c\left({ }^{\prime} K\right)$ $=\alpha^{2} S c(K),{ }^{\prime} \nabla_{i}=\nabla_{\imath},{ }^{\prime} \nabla^{\imath}=\alpha^{2} \nabla^{i},{ }^{\prime} K_{\imath}{ }^{h}=\alpha^{2} K_{\imath}{ }^{h},{ }^{\prime} K_{\jmath q p \imath}{ }^{\prime} K^{q p}=\alpha^{2} K_{\jmath q p i} K^{q p},{ }^{\prime} K^{r q p}{ }_{\jmath}{ }^{\prime} K_{r q p \imath}$ $=\alpha^{2} K^{r q p}{ }_{j} K_{r q p \imath},{ }^{\prime} K_{d c b a} K^{d c b a}=\alpha^{4} K_{d c b a} K^{d c b a}$. Hence we get

$$
\begin{aligned}
2^{\prime} \nabla_{\jmath}{ }^{\prime} & \nabla_{i} S c\left({ }^{\prime} K\right)-4^{\prime} \nabla_{p}{ }^{\prime} \nabla^{p \prime} K_{j i} \\
& +4^{\prime} K_{\jmath p}{ }^{\prime} K^{p}{ }_{i}-4^{\prime} K_{\jmath q p \imath}{ }^{\prime} K^{q p} \\
& -2^{\prime} K^{r q p}{ }_{j}^{\prime} K_{r q p i}+\frac{1}{2}^{\prime} K_{d c b a}{ }^{\prime} K^{d c b a}{ }^{\prime} g_{j i}=c \alpha^{4} g_{j i}
\end{aligned}
$$

where $c$ is the same number as in (2.2).

As $c$ is not given beforehand, we get the following lemma.

LEMMA 2.1. Let $M$ be a compact ornentable $C^{\infty}$ manifold and ' $g$ be a $C^{\infty}$ Riemannian metric on $M$. A necessary and sufficient condition that there exist a critucal Remannian metric $g$ homothetuc to ' $g$ is that there exist a constant $c_{1}$ such that

$$
\begin{aligned}
2^{\prime} \nabla_{\jmath}{ }^{\prime} & \nabla_{i} S c\left({ }^{\prime} K\right)-4^{\prime} \nabla_{p}{ }^{\prime} \nabla^{p \prime} K_{j i} \\
& +4^{\prime} K_{\jmath p}{ }^{\prime} K^{p}{ }_{i}-4^{\prime} K_{\jmath q p \imath}{ }^{\prime} K^{q p} \\
& -2^{\prime} K^{r q p}{ }_{\jmath}{ }^{\prime} K_{r q p i}+\frac{1}{2}{ }^{\prime} K_{d c b a}{ }^{\prime} K^{d c b a}{ }^{\prime} g_{j i}=c_{1}{ }^{\prime} g_{j i}
\end{aligned}
$$

Now let us return to the subject and prove Theorem 1 .

A necessary and sufficient condition that there exist a critical Riemannian metric $g_{1}$ on $M_{1}$ such that $g_{1}=\left(\alpha_{1}\right)^{2} g_{1}$, where $\alpha_{1}$ is a positive number, is, as we see immediately from Lemma 2.1, that there exist a constant $c_{1}$ such that

$$
\begin{aligned}
2^{\prime} \nabla_{\jmath}{ }^{\prime} \nabla_{i} S c\left({ }^{\prime} K_{1}\right)-4^{\prime} \nabla_{p}{ }^{\prime} \nabla^{p} K_{j i} \\
\quad+4^{\prime} K_{\jmath p} K^{p}{ }_{i}-4^{\prime} K_{\jmath q p \imath}{ }^{\prime} K^{q p} \\
\quad-2^{\prime} K^{r q p}{ }_{\jmath}{ }^{\prime} K_{r q p}+\frac{1}{2}{ }^{\prime} K_{d c b a}{ }^{\prime} K^{d c b a}{ }^{\prime} g_{j i}=c_{1}{ }^{\prime} g_{j i}
\end{aligned}
$$

where all tensors, the scalar curvature $S c\left({ }^{\prime} K_{1}\right)$ and covariant differentiation are those of the Riemannian structure in $\left(M_{1},{ }^{\prime} g_{1}\right)$. Similarly, a necessary and sufficient condition that there exist a critical Riemannian metric $g_{2}$ on $M_{2}$ such that $g_{2}=\left(\alpha_{2}\right)^{2 \prime} g_{2}$ is that there exist a constant $c_{2}$ such that

$$
\begin{aligned}
2^{\prime} \nabla_{\mu}{ }^{\prime} & \nabla_{\lambda} S c\left(K_{2}\right)-4^{\prime} \nabla_{\rho}^{\prime} \nabla^{\rho \prime} K_{\mu \lambda} \\
& +4^{\prime} K_{\mu \rho}{ }^{\prime} K^{\rho}{ }_{\lambda}-4^{\prime} K_{\mu \sigma \rho \lambda}{ }^{\prime} K^{\sigma \rho} \\
& -2^{\prime} K^{\tau \sigma \rho}{ }_{\mu}{ }^{\prime} K_{\tau \sigma \rho \lambda}+\frac{1}{2}^{\prime} K_{\tau \sigma \rho \pi}{ }^{\prime} K^{\tau \sigma \rho \pi \prime} g_{\mu \lambda}=c_{2}{ }^{\prime} g_{\mu \lambda}
\end{aligned}
$$


where all tensors, the scalar curvature $S c\left({ }^{\prime} K_{2}\right)$ and covariant differentiation are those of $\left(M_{2}, g_{2}\right)$.

On the other hand, a necessary and sufficient condition that $g_{12}$ with components $g_{J I}$ be a critical Riemannian metric on $M$ is that there exist a constant $c$ such that

$$
\begin{array}{rl}
2 \nabla_{J} \nabla_{I} & S c(K)-4 \nabla_{P} \nabla^{P} K_{J I} \\
& +4 K_{J P} K^{P_{I}}-4 K_{J Q P I} K^{Q P} \\
& -2 K^{R Q P}{ }_{J} K_{R Q P I}+\frac{1}{2} K_{D C B A} K^{D C B A} g_{J I}=c g_{J I}
\end{array}
$$

where all tensors, the scalar curvature $S c(K)$ and covariant differentiation are those of $\left(M, g_{12}\right)$.

As we have (1.1), all genuine quantities of $\left(M_{1},{ }^{\prime} g_{1}\right)$ do not depend on $x^{\kappa}$ and all genuine quantities of $\left(M_{2},{ }^{\prime} g_{2}\right)$ do not depend on $x^{h}$. From (1.1) and all the formulas following (1.1) we thus obtain following relations between quantities in $\left(M, g_{12}\right)$ and quantities in $\left(M_{1},{ }^{\prime} g_{1}\right)$ or $\left(M_{2},{ }^{\prime} g_{2}\right)$,

$$
\begin{aligned}
& \nabla_{i} S c(K)={ }^{\prime} \nabla_{i} S c\left({ }^{\prime} K_{1}\right), \quad \nabla_{\lambda} S c(K)={ }^{\prime} \nabla_{\lambda} S c\left({ }^{\prime} K_{2}\right), \\
& \nabla_{j} \nabla_{i} S c(K)={ }^{\prime} \nabla_{j}^{\prime} \nabla_{i} S c\left({ }^{\prime} K_{1}\right), \quad \nabla_{\mu} \nabla_{\lambda} S c(K)={ }^{\prime} \nabla_{\mu}^{\prime} \nabla_{\lambda} S c\left({ }^{\prime} K_{2}\right), \quad \nabla_{j} \nabla_{\lambda} S c(K)=0, \\
& \nabla_{P} \nabla^{P} K_{j i}={ }^{\prime} \nabla_{p}^{\prime} \nabla^{p \prime} K_{j i}, \quad \nabla_{P} \nabla^{P} K_{\mu \lambda}={ }^{\prime} \nabla_{\rho}^{\prime} \nabla^{o \prime} K_{\mu \lambda}, \quad \nabla_{P} \nabla^{P} K_{j \lambda}=0, \\
& K_{j P} K^{P_{i}}=^{\prime} K_{j p} K^{p}{ }_{\imath}, \quad K_{\mu P} K^{P_{\lambda}}={ }^{\prime} K_{\mu \rho}{ }^{\prime} K_{\lambda}^{\rho}, \quad K_{j P} K_{\lambda}^{P_{\lambda}}=0 \text {, } \\
& K_{j Q P i} K^{Q P}={ }^{\prime} K_{j q p \imath}{ }^{\prime} K^{q p}, \quad K_{\mu Q P \lambda} K^{Q P}={ }^{\prime} K_{\mu \sigma \rho \lambda}{ }^{\prime} K^{\sigma \rho}, \quad K_{j Q P \lambda} K^{Q P}=0, \\
& K^{R Q P}{ }_{j} K_{R Q P i}={ }^{\prime} K^{r q p}{ }_{j}{ }^{\prime} K_{r q p \imath}, \quad K^{R Q P}{ }_{\mu} K_{R Q P \lambda}={ }^{\prime} K^{\tau \sigma \rho}{ }_{\mu}{ }^{\prime} K_{\tau \sigma \rho \lambda}, \quad K^{R Q P}{ }_{j} K_{R Q P \lambda}=0 \text {, } \\
& K_{D C B A} K^{D C B A}={ }^{\prime} K_{d c b a}{ }^{\prime} K^{d c b a}+{ }^{\prime} K_{\tau \sigma \rho \pi} K^{\tau \sigma \rho \pi} \text {. }
\end{aligned}
$$

Thus (2.7) is equivalent in this case to the following set of equations (2.8) and (2.9),

$$
\begin{aligned}
& 2^{\prime} \nabla_{\jmath}^{\prime} \nabla_{i} S c\left(K_{1}\right)-4^{\prime} \nabla_{p}{ }^{\prime} \nabla^{p \prime} K_{j \imath} \\
& +4^{\prime} K_{\jmath p}{ }^{\prime} K^{p}{ }_{i}-4^{\prime} K_{\partial q p \imath}{ }^{\prime} K^{q p} \\
& -2^{\prime} K^{r q p}{ }^{\prime} K_{r q p \imath}+\frac{1}{2}\left({ }^{\prime} K_{d c b a} K^{d c b a}+{ }^{\prime} K_{\tau \sigma o \sigma^{\prime}} K^{\tau \sigma o \sigma}\right)^{\prime} g_{\jmath i}=c^{\prime} g_{j i}, \\
& 2^{\prime} \nabla_{\mu}^{\prime} \nabla_{\lambda} S c\left(K_{2}\right)-4^{\prime} \nabla_{\rho}^{\prime} \nabla^{\rho \prime} K_{\mu \lambda} \\
& +4^{\prime} K_{\mu \rho}{ }^{\prime} K_{\lambda}^{\rho}-4^{\prime} K_{\mu \sigma \rho \lambda}{ }^{\prime} K^{\sigma \rho} \\
& -2^{\prime} K^{\tau \sigma \rho}{ }_{\mu}^{\prime} K_{\tau \sigma \rho \lambda}+\frac{1}{2}\left({ }^{\prime} K_{d c b a}{ }^{\prime} K^{d c b a}+{ }^{\prime} K_{\tau \sigma \rho \pi^{\prime}} K^{\tau \sigma \rho \pi}\right)^{\prime} g_{\mu \lambda}=c^{\prime} g_{\mu \lambda} \text {. }
\end{aligned}
$$


Now let us assume that $g_{1}=\left(\alpha_{1}\right)^{2 \prime} g_{1}$ and $g_{2}=\left(\alpha_{2}\right)^{2 \prime} g_{2}$ are critical Riemannian metrics on $M_{1}$ and $M_{2}$ respectively. Then we get from (2.5) and (2.8) or from (2.6) and (2.9)

$$
c_{1}+\frac{1}{2}-\left\|^{\prime} K_{2}\right\|^{2}=c, \quad c_{2}+\frac{1}{2}\left\|^{\prime} K_{1}\right\|^{2}=c
$$

where ' $K_{1}$ and ' $K_{2}$ are the curvature tensors of $\left(M_{1},{ }^{\prime} g_{1}\right)$ and $\left(M_{2},{ }^{\prime} g_{2}\right)$ respectively. Thus we have

$$
\left\|^{\prime} K_{1}\right\|^{2}-\left\|^{\prime} K_{2}\right\|^{2}=2\left(c_{1}-c_{2}\right),
$$

which proves that, if $g_{12}, g_{1}$ and $g_{2}$ are critical Riemannian metrics on $M, M_{1}$ and $M_{2}$ respectively, then $\left\|^{\prime} K_{1}\right\|^{2}$ and $\left\|^{\prime} K_{2}\right\|^{2}$ are constant on $M$.

Furthermore, we get from (2.5) and (2.6), by transvecting with ' $g^{j i}$ and ' $g^{\mu \lambda}$,

$$
\begin{aligned}
& c_{1}=-\frac{2}{m_{1}} \nabla_{p}^{\prime} \nabla^{p} S c\left(^{\prime} K_{1}\right)+\left(\frac{1}{2}-\frac{2}{m_{1}}\right)\left\|^{\prime} K_{1}\right\|^{2}, \\
& c_{2}=-\frac{2}{m_{2}} \nabla_{\rho}^{\prime} \nabla^{\rho} S c\left(^{\prime} K_{2}\right)+\left(\frac{1}{2}-\frac{2}{m_{2}}\right)\left\|^{\prime} K_{2}\right\|^{2} .
\end{aligned}
$$

Hence ${ }^{\prime} \nabla_{p}^{\prime} \nabla^{p} S c\left(K_{1}\right)$ must be constant. But we have

$$
\int_{M_{1}}{ }^{\prime} \nabla_{p}^{\prime} \nabla^{p} S c\left({ }^{\prime} K_{1}\right) d V_{g_{1}}=0 \text {. }
$$

Thus we get $S c\left({ }^{\prime} K_{1}\right)=$ const. Similarly we get $S c\left({ }^{\prime} K_{2}\right)=$ const. At the same time we get

$$
c_{1}=\left(\frac{1}{2}-\frac{2}{m_{1}}\right)\left\|^{\prime} K_{1}\right\|^{2}, \quad c_{2}=\left(\frac{1}{2}-\frac{2}{m_{2}}\right)\left\|^{\prime} K_{2}\right\|^{2} .
$$

From this and (2.11) we get

$$
\frac{\left\|^{\prime} K_{1}\right\|^{2}}{m_{1}}=\frac{\left\|^{\prime} K_{2}\right\|^{2}}{m_{2}}
$$

and

$$
\frac{\left(\alpha_{1}\right)^{4}\left\|K_{1}\right\|^{2}}{m_{1}}=\frac{\left(\alpha_{2}\right)^{4}\left\|K_{2}\right\|^{2}}{m_{2}} .
$$

Conversely, if we have (2.14) where $\left\|^{\prime} K_{1}\right\|^{2}$ and $\left\|^{\prime} K_{2}\right\|^{2}$ are constant, then we have ${ }^{\prime} \nabla_{p}^{\prime} \nabla^{p} S c\left({ }^{\prime} K_{1}\right)=$ const and ${ }^{\prime} \nabla_{\rho}^{\prime} \nabla^{\rho} S c\left({ }^{\prime} K_{2}\right)=$ const from (2.12), hence $S c\left({ }^{\prime} K_{1}\right)$ $=$ const and $S c\left({ }^{\prime} K_{2}\right)=$ const. Thus we get (2.13). Furthermore we can determine $c$ by (2.10). As we have (2.5) and (2.6), we get (2.8) and (2.9).

Thus we have proved Theorem 1.

From this theorem we get

THEOREM 2.2. Let $M, M_{1}, M_{2}$ be compact orientable $C^{\infty}$ manifolds such that $M=M_{1} \times M_{2}$. Assume that $g_{1}$ and $g_{2}$ are non-flat critical Riemannian metrics on $M_{1}$ and $M_{2}$ respectively. Then a necessary and sufficient condition that there exist $a$ critical Riemannian metric $g_{12}$ on $M$ and Riemannian metrics ' $g_{1}$ and ' $g_{2}$ 
satısfying

$$
\left(M, g_{12}\right)=\left(M_{1}, g_{1}\right) \times\left(M_{2}, g_{2}\right)
$$

and such that ' $g_{1}$ and ' $g_{2}$ are homothetic to $g_{1}$ and $g_{2}$ respectively is that the squares of the curvature tensors, $\left\|K_{1}\right\|^{2}$ and $\left\|K_{2}\right\|^{2}$, of $\left(M_{1}, g_{1}\right)$ and $\left(M_{2}, g_{2}\right)$ be constant.

Proof. If such a critical Riemannian metric $g_{12}$ exists and if we put $' g_{1}=\alpha_{1}{ }^{-2} g_{1}$ and ${ }^{\prime} g_{2}=\alpha_{2}{ }^{-2} g_{2}$, we get $\left\|^{\prime} K_{1}\right\|^{2}=\alpha_{1}{ }^{4}\left\|K_{1}\right\|^{2},\left\|^{\prime} K_{2}\right\|^{2}=\alpha_{2}{ }^{4}\left\|K_{2}\right\|^{2}$. Thus $\left\|K_{1}\right\|^{2}$ and $\left\|K_{2}\right\|^{2}$ are constant because of Theorem 1 . Conversely let us assume $\left\|K_{1}\right\|^{2}$ and $\left\|K_{2}\right\|^{2}$ are constant. If we put

$$
m_{2}\left\|K_{1}\right\|^{2}=m_{1}\left\|K_{2}\right\|^{2} A^{4\left(m_{1}+m_{2}\right)},
$$

4 is constant and does not vanish as $g_{1}$ and $g_{2}$ are non flat. Then

$$
\text { ' } g_{1}=\alpha_{1}{ }^{-2} g_{1}, \quad ' g_{2}=\alpha_{2}{ }^{-2} g_{2}
$$

where

$$
\alpha_{1}=A^{-m_{2}}, \quad \alpha_{2}=A^{m_{1}}
$$

are Riemannian metrics such that

hence

$$
\left\|^{\prime} K_{1}\right\|^{2}=A^{-4 m_{2}}\left\|K_{1}\right\|^{2}, \quad\left\|^{\prime} K_{2}\right\|^{2}=A^{4 m_{1}}\left\|K_{2}\right\|^{2},
$$

$$
\frac{\left\|K_{1}\right\|^{2}}{m_{1}}=\frac{\left\|^{\prime} K_{2}\right\|^{2}}{m_{2}}
$$

Moreover $g_{12} \in \mathscr{M}(M)$ because of $\alpha_{1}^{m_{1}} \alpha_{2}{ }^{m_{2}}=1$. Thus $g_{12}$ is a critical Riemannian metric because of Theorem 1 .

\section{§3. Proof of Theorem 2 .}

Next let us consider the case in which $g_{12},{ }^{\prime} g_{1}$ and ' $g_{2}$ satisfy (1.1) and $g_{12}$ is a critical Riemannian metric on $M$. Then we get from (2.8) and (2.9)

$$
\begin{aligned}
& -2^{\prime} \nabla_{p}^{\prime} \nabla^{p} S c\left(K_{1}\right)-2\left\|^{\prime} K_{1}\right\|^{2}+\frac{m_{1}}{2}\left(\left\|^{\prime} K_{1}\right\|^{2}+\left\|^{\prime} K_{2}\right\|_{1}^{\prime 2}\right)=m_{1} c, \\
& -2^{\prime} \nabla_{\rho}^{\prime} \nabla^{\circ} S c\left({ }^{\prime} K_{2}\right)-2\left\|^{\prime} K_{2}\right\|^{2}+\frac{m_{2}}{2}-\left(\left\|^{\prime} K_{1}\right\|^{2}+\left\|^{\prime} K_{2}\right\|^{2}\right)=m_{2} c .
\end{aligned}
$$

Hence

$$
\begin{aligned}
\frac{1}{m_{1}}\left\{{ }^{\prime} \nabla_{p}{ }^{\prime} \nabla^{p} S c\left({ }^{\prime} K_{1}\right)+\left\|^{\prime} K_{1}\right\|^{2}\right\} & =\frac{1}{m_{2}}\left\{{ }^{\prime} \nabla_{\rho}^{\prime} \nabla^{o} S c\left({ }^{\prime} K_{2}\right)+\left\|^{\prime} K_{2}\right\|^{2}\right\} \\
& =\frac{1}{2}\left[\frac{1}{2}\left(\left\|^{\prime} K_{1}\right\|^{2}+\left\|^{\prime} K_{2}\right\|^{2}\right)-c\right]
\end{aligned}
$$

is a constant which we shall write $C$. Then we have 


$$
-2 C+\frac{1}{2}\left(\left\|^{\prime} K_{1}\right\|^{2}+\left\|^{\prime} K_{2}\right\|^{2}\right)=c
$$

and consequently $\left\|^{\prime} K_{1}\right\|^{2},\left\|^{\prime} K_{2}\right\|^{2},{ }^{\prime} \nabla_{p}^{\prime} \nabla^{p} S c\left({ }^{\prime} K_{1}\right),{ }^{\prime} \nabla_{\rho}^{\prime} \nabla^{\circ} S c\left(K_{2}\right)$ are constants on $M$. Thus $S c\left({ }^{\prime} K_{1}\right)$ and $S c\left({ }^{\prime} K_{2}\right)$ are again constants.

On the other hand we get from (2.8)

$$
\begin{array}{r}
2^{\prime} \nabla_{\jmath}{ }^{\prime} \nabla_{i} S c\left({ }^{\prime} K_{1}\right)-4^{\prime} \nabla_{p}{ }^{\prime} \nabla^{p \prime} K_{j i}+4^{\prime} K_{\jmath p} K^{p}{ }_{i}-4^{\prime} K_{\jmath q p i}{ }^{\prime} K^{q p} \\
-2^{\prime} K^{r q p}{ }_{j}^{\prime} K_{r q p i}+\frac{1}{2}\left\|^{\prime} K_{1}\right\|^{2 \prime} g_{j i}=\left\{c-\frac{1}{2}\left\|^{\prime} K_{2}\right\|^{2}\right\}^{\prime} g_{j i}
\end{array}
$$

which is equivalent to (2.6) if we put

$$
c_{1}=c-\frac{1}{2}\left\|^{\prime} K_{2}\right\|^{2} .
$$

Taking Lemma 2.1 into account, we see that ' $g_{1}$ is homothetic to a critical Riemannian metric on $M_{1}$. Similarly ' $g_{2}$ is homothetic to a critical Riemannian metric on $M_{2}$. Thus we have proved Theorem 2 in view of Theorem 1.

If $M_{1}$ admits a locally flat Riemannian metric ' $g_{1}$, then we have $\left\|^{\prime} K_{1}\right\|^{2}=0$. Hence (2.14) is not satisfied if $\left\|^{\prime} K_{2}\right\|^{2}>0$. Thus we obtain

THeOREM 3.1. A Riemannian manrfold $(M, g)=\left(M_{1},{ }^{\prime} g_{1}\right) \times\left(M_{2},{ }^{\prime} g_{2}\right)$ can not be a critical Riemannian manifold if $\left(M_{1},{ }^{\prime} g_{1}\right)$ is locally flat and $\left(M_{2},{ }^{\prime} g_{2}\right)$ is not locally flat.

\section{$\S 4$. The index of a critical Riemannian manifold $\left(M_{1},{ }^{\prime} g_{1}\right) \times\left(M_{2}, ' g_{2}\right)$.}

Let $M, M_{1}, M_{2}$ be the same as before and $g_{12}$ in $\left(M, g_{12}\right)=\left(M_{1},{ }^{\prime} g_{1}\right) \times\left(M_{2},{ }^{\prime} g_{2}\right)$ be a critical Riemannian metric. By Theorem $2{ }^{\prime} g_{1}$ and ' $g_{2}$ are homothetic to $g_{1}$ and $g_{2}$ respectively which are critical Riemannian metrics on $M_{1}$ and $M_{2}$ respectively with constant $S c\left(K_{1}\right), S c\left(K_{2}\right),\left\|K_{1}\right\|^{2}$ and $\left\|K_{2}\right\|^{2}$. We examine now the index of $I: \mathscr{M}(M) \rightarrow \boldsymbol{R}$ at the critical point $g_{12}$, which we call the index of the critical Riemannian manifold $\left(M_{1},{ }^{\prime} g_{1}\right) \times\left(M_{2}, ' g_{2}\right)$.

We do not calculate the exact value of this index, but intend to show that in certain cases the index is positive.

Let us take Riemannian metrics $g$ on $M=M_{1} \times M_{2}$ such that the components $g_{J I}$ of $g$ are given by

$$
g_{j i}=e^{2 a(y) \prime} g_{j i}, \quad g_{\mu \lambda}={ }^{\prime} g_{\mu \lambda}, \quad g_{j \lambda}=0
$$

where ' $g_{j i}$ and ' $g_{\mu \lambda}$ are respectively components of ' $g_{1}$ and ' $g_{2}$ again and $a(y)$ is a function of $x^{\kappa}$ only $\left(\kappa=m_{1}+1, \cdots, m_{1}+m_{2}\right)$. that

As $g_{12} \in \mathscr{M}(M)$, in order to maintain the relation $g \in \mathscr{M}(M)$, we take $a(y)$ such

$$
\int_{M} e^{m_{1} a} d V_{g_{12}}=1
$$


Hence, if $d V_{2}$ is the volume element of $M_{2}$ measured by ' $g_{2}$, or $g_{2}$ homothetic to $' g_{2}$, we have

$$
\int_{M_{2}} e^{m_{1} a} d V_{2}=\int_{M_{2}} d V_{2} .
$$

We denote in $\S 4$ the Christoffel symbols obtained from $g$ by $\left\{\begin{array}{l}H \\ J I\end{array}\right\}$, while $'\left\{\begin{array}{c}h \\ j i\end{array}\right\}$ and ' $\left\{\begin{array}{c}\kappa \\ \mu \lambda\end{array}\right\}$ are the same as defined in $\S 1$. Then the Christoffel symbols $\left\{\begin{array}{c}H \\ J I\end{array}\right\}$, written separately as $\left\{\begin{array}{c}h \\ \jmath \imath\end{array}\right\},\left\{\begin{array}{c}\kappa \\ j i\end{array}\right\}$ and so on, satisfy the following equations,

$$
\begin{aligned}
& \left\{\begin{array}{c}
h \\
j i
\end{array}\right\}=\left\{\begin{array}{c}
h \\
j i
\end{array}\right\}, \\
& \left\{\begin{array}{c}
\kappa \\
j i
\end{array}\right\}=\frac{1}{2} g^{\kappa \tau}\left(-\partial_{\tau} g_{j i}\right)=-g_{j i}{ }^{\prime} \nabla^{\kappa} a, \\
& \left\{\begin{array}{c}
h \\
j \lambda
\end{array}\right\}=\frac{1}{2} g^{h p} \partial_{\lambda} g_{\jmath p}=\delta_{\jmath}{ }^{h} \partial_{\lambda} a=\delta_{\jmath}{ }^{\prime} \nabla_{\lambda} a, \\
& \left\{\begin{array}{c}
\kappa \\
j \lambda
\end{array}\right\}=0, \quad\left\{\begin{array}{c}
h \\
\mu \lambda
\end{array}\right\}=0, \\
& \left\{\begin{array}{c}
\kappa \\
\mu \lambda
\end{array}\right\}=\left\{\begin{array}{c}
\kappa \\
\mu \lambda
\end{array}\right\},
\end{aligned}
$$

where ${ }^{\prime} \nabla_{\lambda}$ means covariant differentiation in $\left(M_{2},{ }^{\prime} g_{2}\right)$ and ${ }^{\prime} \nabla^{\kappa}={ }^{\prime} g^{\kappa \lambda /} \nabla_{\lambda}=g^{\kappa \lambda /} \nabla_{\lambda}$.

From these equations we can calculate the components of the curvature tensor of $(M, g)$ and get $^{1)}$

$$
\begin{aligned}
& K_{k j i}{ }^{n}={ }^{\prime} K_{k j \imath}{ }^{n}-{ }^{\prime} \nabla_{\rho} a^{\prime} \nabla^{o} a\left(\delta_{k}{ }^{h} g_{j \imath}-\delta_{j}{ }^{h} g_{k \imath}\right), \\
& K_{\nu j i}{ }^{\kappa}=-\left(\nabla_{\nu}{ }^{\prime} \nabla^{\kappa} a+{ }^{\prime} \nabla_{\nu} a^{\prime} \nabla^{\kappa} a\right) g_{j i}, \\
& K_{\nu \mu \lambda}{ }^{\kappa}={ }^{\prime} K_{\nu \mu \lambda{ }^{\kappa},} \\
& K_{k j \imath}{ }^{\kappa}=0, \quad K_{k j \lambda}{ }^{\kappa}=0, \quad K_{\nu \mu \imath}{ }^{\kappa}=0 .
\end{aligned}
$$

Furthermore we get

$$
\begin{aligned}
K_{K J I H} K^{K J I H}= & K_{k j i h} K^{k j i n}+4 K_{\nu j i \kappa} K^{\nu j \imath \kappa}+K_{\nu \mu \lambda \kappa} K^{\nu \mu \lambda \kappa} \\
= & e^{-4 a \prime} K_{k j i n}{ }^{\prime} K^{k j i h}-4 e^{-2 a} S c\left({ }^{\prime} K_{1}\right)^{\prime} \nabla_{\rho} a^{\prime} \nabla^{o} a \\
& +2 m_{1}\left(m_{1}-1\right)\left({ }^{\prime} \nabla_{\rho} a^{\prime} \nabla^{\rho} a\right)^{2} \\
& +4 m_{1}\left({ }^{\prime} \nabla_{\mu}^{\prime} \nabla_{\lambda} a+{ }^{\prime} \nabla_{\mu} a^{\prime} \nabla_{\lambda} a\right)\left({ }^{\prime} \nabla^{\mu \prime} \nabla^{\lambda} a+{ }^{\prime} \nabla^{\mu} a^{\prime} \nabla^{\lambda} a\right) \\
& +{ }^{\prime} K_{\nu \mu \lambda \kappa} K^{\nu \mu \lambda \kappa} .
\end{aligned}
$$

1) In these formulas $\nabla A \nabla B$ always means $(\nabla A)(\nabla B)$. 

(4.2)

hence

Let us assume $|a|$ to be so small that we can neglect $a^{3}$. Then we get from

$$
\int_{M_{2}} a d V_{2}=-\frac{m_{1}}{2} \int_{M_{2}} a^{2} d V_{2}
$$

$$
\int_{M} a d V_{g_{12}}=-\frac{m_{1}}{2} \int_{M} a^{2} d V_{g_{12}}
$$

Consequently we have

$$
\begin{aligned}
\int_{M} K_{K J I H} K^{K . J I H} d V_{g}=\int_{M} & {\left[K_{k j i h}{ }^{\prime} K^{k j i n}\left\{1+\left(m_{1}-4\right) a+\frac{\left(m_{1}-4\right)^{2}}{2} a^{2}\right\}\right.} \\
& -4 S c\left({ }^{\prime} K_{1}\right)^{\prime} \nabla_{\rho} a^{\prime} \nabla^{o} a+4 m_{1}{ }^{\prime} \nabla_{\prime \prime}^{\prime} \nabla_{\lambda} a^{\prime} \nabla^{\prime \prime} \nabla^{\lambda} a \\
& \left.+{ }^{\prime} K_{\nu \mu \lambda \kappa}{ }^{\prime} K^{\nu \mu \lambda \hbar} e^{m_{1} a}\right] d V_{g_{12}}
\end{aligned}
$$

where we have neglected $a^{3}$.

Let us denote this integral by $J[a]$. Then, as $S c\left({ }^{\prime} K_{1}\right),\left\|^{\prime} K_{1}\right\|^{2}$ and $\left\|^{\prime} K_{2}\right\|^{2}$ are constant, we get

$$
\begin{aligned}
J[a]-J[0]= & -2\left(m_{1}-4\right)\left\|^{\prime} K_{1}\right\|^{2} \int_{M} a^{2} d V \\
& -4 S c\left({ }^{\prime} K_{1}\right) \int_{M}{ }^{\prime} \nabla_{\rho} a^{\prime} \nabla^{\rho} a d V+4 m_{1} \int_{M}{ }^{\prime} \nabla_{\mu}^{\prime} \nabla_{\lambda} a^{\prime} \nabla^{\mu \prime} \nabla^{\lambda} a d V
\end{aligned}
$$

where $d V$ is the volume element of $M$ measured by $g_{12}$.

Let $f(y)$ be a function on $M_{2}$ satisfying

$$
{ }^{\prime} \nabla_{\mu}^{\prime} \nabla^{\mu} f=-\lambda_{1} f, \quad \int_{M_{2}} f^{2} d V_{2}=1
$$

where $\lambda_{1}$ is the smallest positive eigenvalue of the Laplacian. If $\alpha$ is a small positive number and

$$
a(y)=\alpha f(y)-\frac{m_{1}}{2} \alpha^{2}(f(y))^{2},
$$

we get

$$
\int_{M_{2}} a d V_{2}=-\frac{m_{1}}{2} \alpha^{2}=-\frac{m_{1}}{2} \int_{M_{2}} a^{2} d V_{2}
$$

neglecting $a^{3}$. In this case we have

$$
\begin{aligned}
J[a]-J[0]= & {\left[\left\{-2\left(m_{1}-4\right)\left\|^{\prime} K_{1}\right\|^{2}-4 \lambda_{1} S c\left({ }^{\prime} K_{1}\right)\right\}\right.} \\
& \left.+4 m_{1} \int_{M} \nabla_{\mu}^{\prime} \nabla_{\lambda} f^{\prime} \nabla^{\mu \prime} \nabla^{\lambda} f d V\right] \alpha^{2},
\end{aligned}
$$

or, if we use

$$
\int_{M}{ }^{\prime} \nabla_{\mu}^{\prime} V_{\lambda} a^{\prime} V^{\mu \prime} \nabla^{\lambda} a d V=\int_{M}\left({ }^{\prime} \nabla_{\mu}^{\prime} V^{\mu} a\right)^{2} d V-\int_{M}{ }^{\prime} K^{\mu \lambda \prime} \nabla_{\mu} a^{\prime} \nabla_{\lambda} a d V,
$$


then

$$
\begin{aligned}
J[a]-J[0]=[\{ & \left.-2\left(m_{1}-4\right)\left\|^{\prime} K_{1}\right\|^{2}-4 \lambda_{1} S c\left({ }^{\prime} K_{1}\right)\right\} \\
& \left.+4 m_{1} \lambda_{1}{ }^{2}-4 m_{1} \int_{M}{ }^{\prime} K^{\mu \lambda} \nabla_{\mu} f^{\prime} \nabla_{\lambda} f d V\right] \alpha^{2} .
\end{aligned}
$$

Thus we have the following lemma.

Lemma 4.1. Let $\left(M, g_{12}\right)=\left(M_{1},{ }^{\prime} g_{1}\right) \times\left(M_{2},{ }^{\prime} g_{2}\right)$ be a critical Riemannian manifold and let $\lambda_{1}$ be the smallest positve eigenvalue of the Laplacian on $\left(M_{2},{ }^{\prime} g_{2}\right)$. Let $f$ be an eigenfunction satısfying

$$
\int_{M_{2}} f^{2} d V_{2}=1
$$

If, in this case,

$$
\begin{aligned}
& -2\left(m_{1}-4\right)\left\|^{\prime} K_{1}\right\|^{2}-4 \lambda_{1} S c\left({ }^{\prime} K_{1}\right) \\
& \quad+4 m_{1} \lambda_{1}{ }^{2}-4 m_{1} \int_{M}{ }^{\prime} K^{\mu \lambda} \nabla_{\mu} f^{\prime} \nabla_{\lambda} f d V
\end{aligned}
$$

is negative, the index of the Riemannian manifold $\left(M, g_{12}\right)$ is positive.

COROLlary 4.2. Let the Riemannian manifolds $\left(M, g_{12}\right),\left(M_{1},{ }^{\prime} g_{1}\right),\left(M_{2},{ }^{\prime} g_{2}\right)$, the number $\lambda_{1}$ and the function $f$ be as in Lemma 4.1. Furthermore let $\left(M_{2},{ }^{\prime} g_{2}\right)$ be an Einstern manifold. If, in this case,

$$
-2\left(m_{1}-4\right)\left\|^{\prime} K_{1}\right\|^{2}-4 \lambda_{1} S c\left({ }^{\prime} K_{1}\right)+4 m_{1} \lambda_{1}{ }^{2}-4 m_{1} \lambda_{1} \frac{S c\left({ }^{\prime} K_{2}\right)}{m_{2}}
$$

is negatıve, the index of the Riemannian manifold $\left(M, g_{12}\right)$ is positıve.

\section{$\S 5$. The index of a critical Riemannian manifold $\left(M, g_{12}\right)=\left(M_{1}, g_{1}\right) \times\left(M_{2}, g_{2}\right)$ where $M_{2}$ is a sphere.}

Let us consider a critical Riemannian manifold

$$
\left(M, g_{12}\right)=\left(M_{1}, ' g_{1}\right) \times\left(S, g_{2}\right)
$$

where $S$ is an $m_{2}$-sphere and ' $g_{2}$ is a Riemannian metric of constant curvature with $S c\left({ }^{\prime} K_{2}\right)>0$. Then we have

$$
\lambda_{1}=\frac{S c\left({ }^{\prime} K_{2}\right)}{m_{2}-1}
$$

and there exists a function $f$ on $S$ satısfying

$$
{ }^{\prime} \nabla_{\mu}^{\prime} \nabla_{\lambda} f=-\frac{S c\left({ }^{\prime} K_{2}\right)}{m_{2}\left(m_{2}-1\right)} f^{\prime} g_{\mu \lambda} .
$$

In this case we get 
because of

$$
\begin{aligned}
J[a]-J[0]=[ & -2\left(m_{1}-4\right)\left\|^{\prime} K_{1}\right\|^{2}-4 \frac{S c\left({ }^{\prime} K_{1}\right) S c\left({ }^{\prime} K_{2}\right)}{m_{2}-1} \\
& \left.+4 \frac{m_{1}\left(S c\left({ }^{\prime} K_{2}\right)\right)^{2}}{\left(m_{2}-1\right)^{2}}-4 \frac{m_{1}\left(S c\left({ }^{\prime} K_{2}\right)\right)^{2}}{m_{2}\left(m_{2}-1\right)}\right] \alpha^{2}
\end{aligned}
$$

$$
{ }^{\prime} K^{\mu \lambda}=\frac{1}{m_{2}} S c\left({ }^{\prime} K_{2}\right)^{\prime} g^{\mu \lambda} .
$$

On the other hand we have (2.14) where we can put

$$
\left\|^{\prime} K_{2}\right\|^{2}=\frac{2\left(S c\left({ }^{\prime} K_{2}\right)\right)^{2}}{m_{2}\left(m_{2}-1\right)}
$$

Consequently we get

$$
\begin{aligned}
& J[a]-J[0] \\
& \quad=\left[4 m_{1} \frac{-\left(m_{1}-4\right)\left(m_{2}-1\right)+m_{2}}{m_{2}{ }^{2}\left(m_{2}-1\right)^{2}}\left(S c\left(^{\prime} K_{2}\right)\right)^{2}-4 \frac{S c\left({ }^{\prime} K_{1}\right) S c\left({ }^{\prime} K_{2}\right)}{m_{2}-1}\right] \alpha^{2} .
\end{aligned}
$$

Thus we have proved the following theorem.

THEOREM 5.1. Let $\left(M, g_{12}\right)$ be a critical Riemannian manifold such that

$$
\left(M, g_{12}\right)=\left(M_{1},{ }^{\prime} g_{1}\right) \times\left(S,{ }^{\prime} g_{2}\right)
$$

where $S$ is an $m_{2}$-sphere and ' $g_{2}$ is a Riemannian metric of constant curvature with $S c\left({ }^{\prime} K_{2}\right)>0$. If $S c\left({ }^{\prime} K_{1}\right) \geqq 0$ and $m_{1}$ and $m_{2}$ are such that

$$
\left(m_{1}-4\right)\left(m_{2}-1\right)-m_{2}>0,
$$

then the index of this critucal Riemannian manifold is positive. If $m_{1}=4$ and

$$
S c\left({ }^{\prime} K_{1}\right)>\frac{4 S c\left({ }^{\prime} K_{2}\right)}{m_{2}\left(m_{2}-1\right)},
$$

then the index of $\left(M, g_{12}\right)$ is also positive.

We can also prove the following theorem.

TheOREM 5.2. Let $g_{12}$ be a critical Riemannıan metric on $S_{1} \times S_{2}$ such that

$$
\left(S_{1} \times S_{2}, g_{12}\right)=\left(S_{1}, g_{1}\right) \times\left(S_{2}, ' g_{2}\right)
$$

where $S_{1}$ is an $m_{1}$-sphere and $S_{2}$ is an $m_{2}$-sphere and each of ' $g_{1}$ and ' $g_{2}$ is a Riemannian metric of positive constant curvature. If $m_{1} \geqq 3$ and $m_{2} \geqq 3$, or, if $m_{1} \geqq 4$ and $m_{2}=2$, the index of $\left(S_{1} \times S_{2}, g_{12}\right)$ is positive.

Proof. As we have

we get

$$
\left\|^{\prime} K_{1}\right\|^{2}=\frac{2\left(S c\left({ }^{\prime} K_{1}\right)\right)^{2}}{m_{1}\left(m_{1}-1\right)}, \quad\left\|^{\prime} K_{2}\right\|^{2}=\frac{2\left(S c\left({ }^{\prime} K_{2}\right)\right)^{2}}{m_{2}\left(m_{2}-1\right)},
$$




$$
\frac{\left(S c\left({ }^{\prime} K_{1}\right)\right)^{2}}{m_{1}^{2}\left(m_{1}-1\right)}=\frac{\left(S c\left({ }^{\prime} K_{2}\right)\right)^{2}}{m_{2}^{2}\left(m_{2}-1\right)}
$$

Substituting this into (5.1), we get

$$
\begin{aligned}
J[a]-J[0]= & -\frac{4 m_{1}}{m_{2}^{2}\left(m_{2}-1\right)^{2}}\left[\left(m_{1}-4\right)\left(m_{2}-1\right)\right. \\
& \left.+m_{2}\left(\sqrt{m_{1}-1} \sqrt{m_{2}-1}-1\right)\right]\left(\alpha S c\left({ }^{\prime} K_{2}\right)\right)^{2} .
\end{aligned}
$$

From this equation we immediately obtain Theorem 5.2.

Remark 3. If

$$
\left(S^{2} \times S^{2}, g_{12}\right)=\left(S^{2},{ }^{\prime} g_{1}\right) \times\left(S^{2},{ }^{\prime} g_{2}\right)
$$

is a critical Riemannian manifold where ' $g_{1}=^{\prime} g_{2}$ is a Riemannian metric of positive constant curvature, $\left(S^{2} \times S^{2}, g_{12}\right)$ is an Einstein manifold. This exists and by Avez's theorem [1] this is a critical Riemannian manifold with index null.

The author wishes to express his hearty thanks to Prof. M. Berger who kindly informed him of Avez's theorem.

\title{
REFERENCES
}

[1] Avez, A., Applications de la formule de Gauss-Bonnet-Chern aux variétés à quatre dimensions, C. R. Acad. Sc., 256 (1963), 5488-5490.

[2] BERGER, M., Quelques formules de variation pour une structure riemannienne, Ann. scient. Éc. Norm. Sup., $4^{\mathrm{e}}$ série, 3 (1970), 285-294.

[3] Berger, M. And D. Ebin, Some decompositions of the space of symmetric tensors on a Riemannian manıfold, J. of Diff. Geometry, 3 (1969), 379-392.

[4] Ebin, D., The manifolds of Riemannian metrics, Proceedings of the Symposium in Pure Mathematics of the American Mathematical Society, 15, 11-40, American Mathematical Society, Providence, 1970.

[5] Mutō, Y., Curvature and critical Riemannian metric, J. Math. Soc. Japan, 26 (1974), 686-697

\author{
Department of Applied Mathematics \\ FACULTY OF ENGINEERING \\ YoKoHama National UNiversity \\ YOKOHAMA, JAPAN
}

